\title{
Self-Reported Depression and Anxiety are Correlated with Functional Disability in Parkinson's Disease
}

Amanda Still* BPhty

School of Physiotherapy, University of Otago, Dunedin, New Zealand

Leigh Hale $P h D$

Dean, School of Physiotherapy, University of Otago, Dunedin, New Zealand

Nicola Swain $P h D$

Associate Professor, Dunedin School of Medicine, University of Otago, Dunedin, New Zealand

Prasath Jayakaran PhD

Senior Lecturer, School of Physiotherapy, University of Otago, Dunedin, New Zealand

* Honours student at the time of the study

\section{ABSTRACT}

Non-motor symptoms, namely cognitive and affective domain function, may impact the physical functioning and perceived health status of people with Parkinson's disease (PD). The aim of this cross-sectional observational study was to explore the relationship between the severity of non-motor symptoms (cognitive and affective) and physical function in individuals with PD living in the community. The outcome measures were completed in 19 participants diagnosed with PD, with or without affective symptoms and cognitive impairments. The main constructs included in the bivariate statistical analyses were: self-reported non-motor experiences of daily living (Movement Disorder Society - Unified Parkinson's Disease Rating Scale [MDS-UPDRS] Part I); self-reported motor experiences of daily living (MDS-UPDRS Part II); clinician-rated impression of motor symptoms (MDS-UPDRS Part III); motor fluctuations (MDS-UPDRS Part IV); self-reported anxiety and depression symptoms (Hospital Anxiety and Depression Rating Scale [HADS] - a total score comprising sub-scores for "anxiety" [HADS-A] and "depression" [HADS-D]); global cognitive function (Montreal Cognitive Assessment [MoCA]); functional gait and balance performance (Dynamic Gait Index [DGI]); and perceived quality of life (European Quality of Life - Visual Analogue Scale [EQ-VAS]). Significant positive correlations $(p \leq 0.05)$ were observed between the MDS-UPDRS Part II and MDS-UPDRS Part I $(p<0.01)$, HADS, HADS-A, and HADS-D $(p<0.05)$. The Hoehn and Yahr (H \& Y) scale was the only variable to significantly correlate with the DGI $(p<0.01)$. MDS-UPDRS Part III significantly correlated with the $\mathrm{H}$ $\& Y$ scale $(p<0.01)$ and MoCA $(p<0.05)$. HADS-D was the only significant and negative correlate with perceived health status $(p<$ 0.05). A significant relationship was observed between the severities of self-reported depression and anxiety, and physical function in people with PD. The severity of the depression symptom was a significant determinant of perceived health status.

Still, A., Hale, L., Swain, N. \& Jayakaran, P. (2021). Self-reported depression and anxiety are correlated with functional disability in Parkinson's disease. New Zealand Journal of Physiotherapy, 49(1), 40-49. https://doi.org/10.15619/ NZJP/49.1.06

Key Words: Anxiety, Cognitive Impairment, Depression, Gait, Non-Motor Symptoms, Parkinson's Disease, Physical Function

\section{INTRODUCTION}

Parkinson's disease (PD) is a progressive neurological condition, which has increased more than two-fold in the last two decades (Mak et al., 2017; Rocca, 2018). The current global prevalence is estimated at 6.1 million (Mak et al., 2017; Rocca, 2018), and is projected to increase by approximately 770,000 by 2040 (Rossi et al., 2018). In New Zealand, PD is one of the leading neurological causes of disability affecting approximately 210 per 100,000 people (Pitcher et al., 2018).

PD significantly impacts physical function, perceived quality of life (QoL), and health status (Schrag et al., 2000). Gait and balance impairments are reportedly the most frequent motor symptoms that contribute to disability and poor healthrelated quality of life (HR-QoL) in people with PD (GBD 2015 Neurological Disorders Collaborator Group, 2017; GBD 2016 Neurology Collaborators, 2018; Soh et al., 2011). Along with motor symptoms, it has been shown that the presence and severity of non-motor symptoms (NMS), particularly in the early stages of $\mathrm{PD}$, have a greater negative impact on the QoL of people with PD than motor disability (Erro et al., 2016; Prakash et al., 2016). The common NMS shown to be significant determinants of HR-QoL are cognitive impairment, depression, and anxiety (Hinnell et al., 2012; Soh et al., 2011). These NMS have also been reported to influence gait parameters, such as stride/step length, gait velocity, and variability in the PD population (Lord et al., 2011).

While the pathophysiology of gait and balance impairments, and the aforementioned NMS in PD is complex, the loss of dopaminergic neurons in the substantia nigra and the resultant deficiency of dopamine concentration appears to be a common contributing etiology (Clark, 2015; Gilat et al., 2017; Khan et al., 2017; Lewis \& Barker, 2009; Peterson \& Horak, 2016). To 
walk safely and effectively, people with PD must compensate for their PD-related gait and balance impairments, which often demands increased cognitive resources, particularly attentiondemanding executive functions (Gilat et al., 2017). The basal ganglia receives, processes and integrates motor, cognitive and limbic inputs from separate cortico-striatal neuronal networks to coordinate function (Lewis \& Barker, 2009). Dopamine depletion reduces the processing capabilities of the basal ganglia, leading to gait and balance impairments (Clark, 2015; Gilat et al., 2017; Lewis \& Barker, 2009). Furthermore, it has been suggested that these processing resources can be overloaded by increased cognitive demands and affective symptoms, and thus augment the gait and balance impairments (Gilat et al., 2017). Figure 1 illustrates the potential relationship between gait and balance impairments, and cognition and affective symptoms in PD. To our knowledge, this relationship between the severity of NMS and motor symptoms has not been reported and is poorly understood. While NMS in PD includes a wide range of symptoms, in this study, NMS refers to cognitive impairment, depression, and anxiety.

\section{Figure 1}

Potential Inter-Relationship Between Functional Gait and Balance Performance With the Severity of Cognitive Impairment, Depression and Anxiety Symptoms

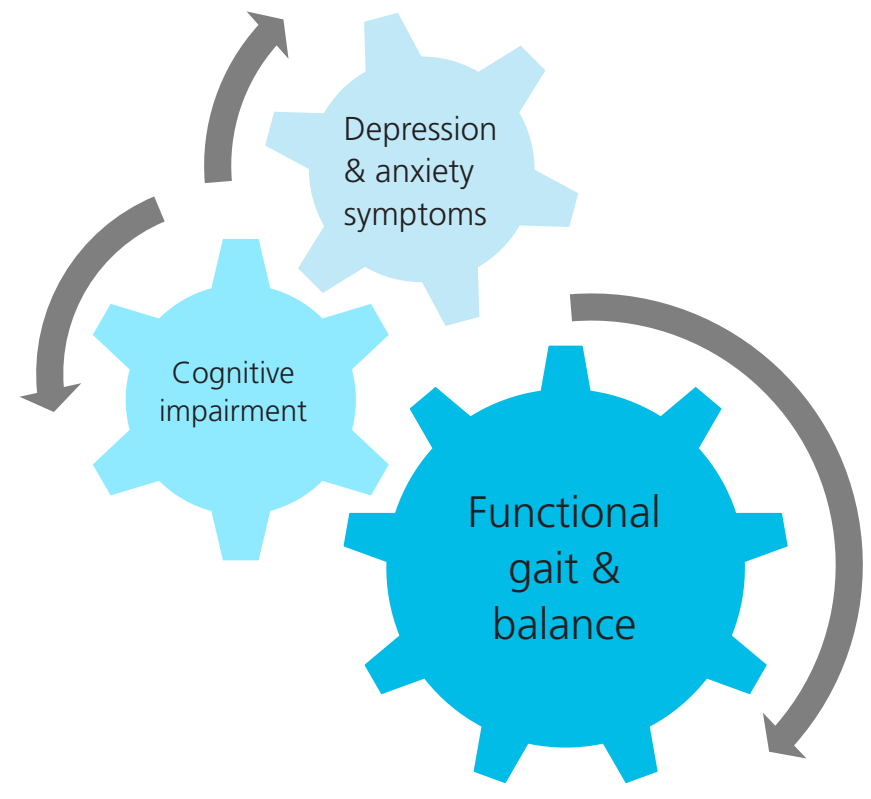

The aim of this study was to gain a preliminary understanding of the relationship between NMS, namely cognitive impairment, depression and anxiety symptoms, with physical function, particularly functional gait and balance performance, and the perceived health status in individuals with PD. In line with the functional pathophysiology of gait and balance impairments in PD, we hypothesised that the severity of these NMS would correlate with motor dysfunction and poorer perceptions of health status. We anticipated an inverse relationship between the increase in severity of cognitive impairment, depression and anxiety symptoms with functional gait and balance performance.

\section{METHODS}

Design

A cross-sectional observational study of participants diagnosed with PD.

\section{Sample size}

In the absence of any current information in the literature about the influence of NMS on physical function, a modest sample size was considered for this exploratory observational study. However, for a bivariate correlation analysis with an expected $r$ $=0.50, \alpha=0.05$, power of $80 \%(\beta=0.2)$, a sample size of $n=$ 20 was estimated for a Spearman rank correlation test (Bonett \& Wright, 2000).

\section{Participants}

Individuals diagnosed with PD were recruited from the local community through flyers placed in the community PD exercise classes, an advertisement in the local newspaper, email correspondence circulated to the members of the local Parkinson's Society, and word of mouth.

The study included individuals diagnosed with PD who could walk at least $100 \mathrm{~m}$ of level ground with or without an ambulatory device. Individuals were excluded from the study if they had co-morbidities, such as vertigo, stroke and arthritis, that could potentially affect their physical function (functional gait and balance). Also excluded were individuals who could not understand and follow instructions and/or who scored $\geq 4$ on item 1.1 of the MDS-UPDRS for cognitive impairment. This set of exclusion criteria was applied to minimise the impact of the other comorbidities on the outcome measures.

\section{Procedure}

Potential participants responding to the recruitment call were provided with a detailed information sheet. Individuals who confirmed their interest to participate were screened for their eligibility over the phone or at the time of assessment. Individuals who had not responded within 2-4 weeks from receiving information about the study were followed-up with an email or a phone call. All participants completed the initial screening and demographic questions comprising age, sex, weight, height, and ethnicity, and time since their diagnosis of PD. Also collected were details of any comorbidities that may have affected their walking ability, or required use of an ambulatory device or support from another person to walk; exercise tolerance; and medications taken for PD. All participants attended one assessment session lasting up to $90 \mathrm{~min}$, with all data recorded on paper forms.

Participants were requested to take their medication as usual, and all outcome measures were administrated during a selfreported "on state". All participants provided written informed consent, and ethical approval was granted by the University of Otago Human Ethics Committee (reference number H19/048).

Outcome measures

The following outcome measures were included in bivariate correlation analysis: Hospital Anxiety and Depression Scale (HADS) - with subscale scores for "anxiety" (HADS-A) and "depression" (HADS-D) (Zigmond \& Snaith, 1983), Montreal Cognitive Assessment (MoCA) (Nasreddine et al., 2005), 
Dynamic Gait Index (DGI) (Herman et al., 2009), Movement Disorder Society - Unified Parkinson's Disease Rating Scale (MDS-UPDRS) Part I-IV (Goetz et al., 2008), and EuroQoL-Visual Analogue Scale (EQ-VAS).

Hospital Anxiety and Depression Scale (HADS) Severity of anxiety and depression symptoms were evaluated using the self-reported HADS, which is reported to be reliable and valid for use in the PD population (Leentjens et al., 2011). HADS-A and HADS-D subscale scores that range from 0 to 21 were calculated to categorize the severity of symptoms (Zigmond \& Snaith, 1983). Each subscale contained seven questions that were scored $0-3$; higher scores denote greater severity. A pre-determined scoring criterion as described elsewhere (Smarr \& Keefer, 2011) was used to interpret the severity of symptoms as follows: $\leq 7$, normal; 8-10; mild; $11-15$, moderate; $\geq 16$, severe (Smarr \& Keefer, 2011) Subscales scores were also summated to give an impression of global mood (Zigmond \& Snaith, 1983).

\section{Montreal Cognitive Assessment (MoCA)}

Global cognitive function was determined using MoCA, which assesses cognitive function across eight domains: executive and visuospatial functions, attention, working memory, language, learning, memory, and orientation (Nasreddine et al., 2005). MoCA is a reliable and valid measure of cognitive function in people with PD (Nasreddine et al., 2005; Skorvanek et al., 2018). Scores from each domain as assigned by the clinician were summated to produce a total score ranging between 0 and 30 (Nasreddine et al., 2005). Scores were interpreted as described elsewhere: normal, $\geq 26$; PD-related mild cognitive impairment, 21-25; PD-related dementia, $\leq 20$ (Skorvanek et al., 2018).

\section{Dynamic Gait Index (DGI)}

The DGI is an eight-item clinician-rated objective assessment of functional gait and balance performance that is a reliable and valid in people with PD (Bloem et al., 2016; Huang et al., 2011). The DGI requires people to adapt their gait during eight different walking demands (Herman et al., 2009). Each item is scored $0-3$ by the clinician, where $0=$ severe impairment, $1=$ moderate impairment, 2 = mild impairment, and $3=$ normal. Scores from each item were summated to produce a score ranging from 0-24; higher scores denote greater functional performance. People who scored $\leq 21$ were interpreted as an increased falls risk (Dibble \& Lange, 2006).

Movement Disorder Society - Unified Parkinson's Disease Rating Scale (MDS-UPDRS) Part I-IV

The MDS-UPDRS is a comprehensive assessment tool of PD-related symptoms and overall disease severity, which is composed of four parts: Part I: Non-motor experiences of daily living (self-reported); Part II: Motor experiences of daily living (self-reported); Part III: Motor examination; and Part IV: Motor complications (Goetz et al., 2008). Each question is scored using a 0 to 4 rating system that is designed to capture both the presence and severity of symptoms where $0=$ normal, 1 = slight, 2 = mild, $3=$ moderate, and $4=$ severe problems (Abdolahi et al., 2013; Goetz et al., 2007; Goetz et al., 2008). Each part was scored separately where higher scores denoted greater disease severity. The disease stage was scored using the
Hoehn and Yahr (H \& Y) five-point ordinal scale from 0-4, with higher scores indicating greater disability (Goetz et al., 2008). The MDS-UPDRS, including the five-point $\mathrm{H} \& \mathrm{Y}$ scale, is reliable and valid for use in the PD population to evaluate disease severity and disability (Goetz et al., 2004; Goetz et al., 2008).

EuroQoL-Visual Analogue Scale (EQ-VAS)

EQ-VAS is a self-reported generic measure used to report a person's perception of their health status on a scale from 0 (worst health imaginable) to 100 (best health imaginable), which was used in the correlation analysis. EQVAS has been recommended by the MDS to evaluate HR-QoL in the PD population (Martinez-Martin et al., 2011). The general perception of QoL (European Quality of Life - five domain, fivelevel questionnaire [EQ-5D-5L]) (Martinez-Martin et al., 2011) was only used as a descriptive categorical variable.

Demographic details and self-reported estimates of physical activity levels over the last 7 days (International Physical Activity Questionnaire - version for elderly [IPAQ-E] - short form) (Craig et al., 2003; Heesch et al., 2010) were obtained to describe the characteristics of the study sample.

\section{Data extraction and processing}

All paper-based data were converted into an electronic format in Microsoft@ Excel. Each outcome measure was processed and scored according to their respective scoring protocol. The score for each of the MDS-UPDRS sub-sections was used for statistical analysis. The IPAQ-E was scored as the total metabolic equivalent from all reported activity, estimated as minutes/week (MET-minutes/weeks) in accordance to scoring criteria described elsewhere (Craig et al., 2003). The EQ-5D-5L dimensions were analysed descriptively.

\section{Statistical analyses}

Statistical analyses were performed with the IBM SPSS Statistics 25.0 software. Spearman's correlation analysis was used to assess the bivariate association of non-motor measures (HADS, HADS-A, HADS-D, MoCA, MDS-UPDRS Part I and H\&Y scores) with physical function measures (DGI, MDS-UPDRS Part II, MDSUPDRS Part III, MDS-UPDRS Part IV) and EQ-VAS. The guideline used to interpret the relationship between variables was as follows: $r \geq 0.75$, good to excellent relationship; 0.50-0.75, moderate to good relationship; 0.25-0.50, fair relationship; 0.00-0.25, little or no relationship (Portney \& Watkins, 2015). A $p$-value (two-tailed) $<0.05$ was considered statistically significant. MDS-UPDRS, IPAQ-E, EQ5D-5L and EQ-VAS were descriptively analysed to inform the disease severity, selfreported physical activity levels, and perceived HR-QoL of study participants. An additional bootstrap analysis of 5,000 was conducted between all bivariate variables to validate statistical findings. While bootstrap analysis has a number of different applications (Lai, 2020), it was used in this study to estimate the confidence intervals, with simulation for 5,000 samples.

Descriptive statistical analyses were performed for all demographic and clinical characteristics of the study population, and are shown as mean, standard deviation, and range. All variables included in the statistical analysis underwent normality testing using the Kolmogorov Smirnoff test $(p<0.05$ was not considered normally distributed) (Field, 2009). A test for normality of the data suggested that MDS-UPDRS Part I, MDS- 
UPDRS Part II, MDS-UPDRS Part IV, H\&Y, DGI, and EQ-VAS were not normally distributed; therefore, Spearman's rank-order correlation coefficients were used.

\section{RESULTS}

Participants

The 25 individuals who expressed preliminary interest in participating in the study were provided with the study information sheet and consent form. After the screening process, a sample of 19 participants were eligible, and they completed all outcome measures. Figure 2 illustrates the stepby-step process of participant recruitment and participation.

All participants except one were regularly taking dopaminergic medication, and in addition, four participants were prescribed anti-depressants and/or anxiolytic medications. Participant demographics and descriptive characteristics are presented in Table 1. The summary of EQ-5D-5L measure for participants is as illustrated in Figure 3.

The median physical activity levels of participants quantified as metabolic equivalents (MET-minutes/week) was 2,000 METminutes/week with a range between 192 and 6,906 METminutes/week.

In the EQ-5D dimensions, the majority of study participants reported "no problems" (32\%-74\%) or only "slight problems" (21\%-58\%). Only 5\%-22\% reported moderate to severe problems. No participants reported extreme disability or an inability to perform tasks.

Descriptive analyses of outcome measures Descriptive analyses of outcome measures are summarised in
Table 2. The majority (79\%) of participants were classed as having a "normal" severity of anxiety and depression symptoms, and normal cognitive function (53\%), evaluated with the HADS and MoCA assessments, respectively. No participants were suggested to have moderate-severe affective symptoms or Parkinson's disease-related dementia according to the respective cut-off scores used.

\section{Bivariate correlation analysis}

The results of Spearman's rank-order correlation analysis are shown in Table 3. Simple bivariate correlation analysis suggested that no significant $(p>0.05)$ correlations existed between DGI and self-reported NMS severity as evaluated by the HADS $\left(r_{s}=\right.$ $0.269)$, HADS-A $\left(r_{s}=0.132\right)$, HADS-D $\left(r_{s}=0.239\right)$, MoCA $\left(r_{s}=\right.$ $0.367, p>0.05)$ or MDS-UPDRS Part I $\left(r_{s}=0.029\right)$. Furthermore, self-reported motor disability and clinician-rated severity of motor symptoms as evaluated by the MDS-UPDRS Part II and MDS-UPDRS Part III did not show any significant correlation with DGI scores. Bootstrap analysis of 5,000 did not change the significance of the results or the confidence intervals. The $H \& Y$ score was found to correlate significantly and negatively with the DGI scores $\left(r_{s}=-0.507, p<0.05\right)$, and positively with disease duration $\left(r_{s}=0.482, p<0.05\right)$ and MDS-UPDRS Part III $\left(r_{s}=0.590, p<0.05\right)$, but disease duration did not correlate significantly with the DGI scores $\left(r_{s}=-0.441, p>0.05\right)$.

Though NMS measures did not correlate significantly with the DGI scores, MDS-UPDRS Part I $\left(r_{s}=0.624\right), \operatorname{HADS}\left(r_{s}=0.575\right)$, HADS-A $\left(r_{s}=0.536\right)$, and HADS-D $\left(r_{s}=0.481\right)$ were shown to correlate significantly $(p<0.05)$ and positively with selfreported motor disability as evaluated by the MDS-UPDRS Part II. Contrastingly, cognitive function as evaluated by MoCA was

\section{Table 1}

Demographic and Clinical Characteristics of Participants

\begin{tabular}{|c|c|c|}
\hline Characteristics & Mean (SD) a & Range (min-max) \\
\hline Age (years) & $68.8(6.5)$ & $55-77$ \\
\hline Sex (male/female) ${ }^{b}$ & $11 / 8$ & \\
\hline Body height $(\mathrm{m})$ & $1.68(0.10)$ & $1.50-1.83$ \\
\hline Body mass (kg) & $74.65(12.24)$ & $53-100$ \\
\hline Disease duration ( years) & $3.77(2.83)$ & $0.92-10$ \\
\hline Education $\geq 12$ years $^{b}$ & 19 & \\
\hline \multicolumn{3}{|l|}{ Number of participants receiving: } \\
\hline PD medication ${ }^{b}$ & 18 & \\
\hline Mood medication ${ }^{\mathrm{b}}$ & 5 & \\
\hline IPAQ-elderly (MET-min/week) & $2,274(1,643)$ & $192-6,906$ \\
\hline Low $^{b}$ & 2 & \\
\hline Moderate $^{b}$ & 13 & \\
\hline High b & 4 & \\
\hline
\end{tabular}

Note. IPAQ-elderly = International Physical Activity Questionnaire - version for elderly; MET = metabolic equivalent; min-max = minimum to maximum; PD = Parkinson's disease.

a Except where indicated; ${ }^{b}$ Number of participants. 


\section{Figure 2}

Participant Recruitment and Retention in the Study



\section{Figure 3}

Summary of the ED-5D-5L Measure for Participants




Table 2

Descriptive Statistics of the Outcome Measures

\begin{tabular}{|c|c|c|}
\hline Outcome measures & Mean $(S D)^{a}$ & Range (min-max) \\
\hline MDS-UPDRS Part I (0-52) & $10.63(6.92)$ & $0-26$ \\
\hline MDS-UPDRS Part II (0-52) & $11.26(7.00)$ & $1-25$ \\
\hline MDS-UPDRS Part III (0-132) & $22.21(10.79)$ & $4-45$ \\
\hline MDS-UPDRS Part IV (0-24) & $3.05(3.32)$ & $0-11$ \\
\hline H \& Y scale (0-5) & $1.79(0.85)$ & $1-4$ \\
\hline HADS (0-42) & $9.32(5.04)$ & $0-16$ \\
\hline HADS-D (0-21) & $4.79(2.88)$ & $0-10$ \\
\hline Normal $(0-7)^{b}$ & 15 & \\
\hline Mild (8-10) b & 4 & \\
\hline HADS-A (0-21) & $4.53(3.12)$ & $0-9$ \\
\hline Normal $(0-7)^{b}$ & 15 & \\
\hline Mild (8-10) b & 4 & \\
\hline $\operatorname{MoCA}(0-30)$ & $26.11(2.62)$ & $22-30$ \\
\hline Normal $(26-30)^{b}$ & 10 & \\
\hline PD-MCI $(21-25)^{b}$ & 9 & \\
\hline DGI (0-24) & $18.41(3.55)$ & $8-22$ \\
\hline Falls risk $(0-21)^{b}$ & 15 & \\
\hline EQ-VAS (0-100) & $76.84(11.08)$ & $50-95$ \\
\hline
\end{tabular}

Note. DGI = Dynamic Gait Index; EQ-VAS = EuroQoL-Visual Analogue Scale; HADS = Hospital Anxiety and Depression Scale; HADS-D = Depression subscale; HADS-A = Anxiety subscale; H \& Y = Hoehn and Yahr; MDS-UPDRS = Movement Disorder Society - Unified Parkinson's Disease Rating Scale; MDS-UPRDS Part I = non-motor experiences subscale score; MDS-UPRDS Part II = motor experiences subscale score; MDS-UPRDS Part III = motor examination subscale score; MDS-UPRDS Part IV = motor complications subscale score; min-max = minimum to maximum; MoCA = Montreal Cognitive Assessment; PD-MCI = Parkinson's disease-related mild cognitive impairment.

a Except where indicated; b Number of participants.

\section{Table 3}

Spearman's Rank-Order Correlation Coefficients for the Associations Between Non-Motor and Motor-Related Clinical Assessments in Addition to Disease Duration, EQ-VAS and H \& Y Score

\begin{tabular}{lcccccc}
\hline & HADS & HADS-A & HADS-D & MoCA & MD-UPDRS Part I & H \& Y \\
\hline DGI & 0.269 & 0.132 & 0.239 & 0.367 & 0.029 & $-0.507^{* *}$ \\
MDS-UPDRS Part II & $0.575^{*}$ & $0.536^{*}$ & $0.481^{*}$ & -0.010 & $0.624^{* *}$ & -0.117 \\
MDS-UPDRS Part III & -0.044 & -0.301 & 0.320 & $-0.471^{*}$ & -0.318 & $0.590^{* *}$ \\
MDS-UPDRS Part IV & 0.442 & $0.586^{*}$ & 0.183 & 0.301 & 0.438 & -0.253 \\
EQ-VAS & -0.322 & -0.075 & $-0.488^{*}$ & 0.156 & -0.093 & -0.247 \\
Disease duration & 0.104 & 0.160 & 0.042 & 0.086 & 0.064 & $0.482^{*}$ \\
\hline
\end{tabular}

Note. Values are two-tailed pairwise results. DGI = Dynamic Gait Index; EQ-VAS = EuroQoL-Visual Analogue Scale; HADS = Hospital Anxiety and Depression Scale; HADS-D = Depression subscale; HADS-A = Anxiety subscale; H \& Y = Hoehn and Yahr; MoCA = Montreal Cognitive Assessment; MDS-UPDRS = Movement Disorder Society - Unified Parkinson's Disease Rating Scale; MDS-UPRDS Part I = Non-motor experiences subscale score; MDS-UPDRS Part II = motor experiences subscale score; MDS-UPRDS Part III = motor examination subscale score; MDS-UPRDS Part IV = motor complications subscale score.

${ }^{*} p<0.05 ;{ }^{* *} p<0.01$. 
not found to correlate significantly with self-reported motor disability, but did correlate with clinician-rated impressions of motor disability as evaluated by the MDS-UPDRS Part III $\left(r_{s}=-0.471, p<0.05\right)$. Furthermore, HADS-A was found to significantly and positively correlate with motor complications $\left(r_{s}=0.586, p<0.05\right)$, and HADS-D was found to be the only significant determinant of health status as evaluated by the EQ$\operatorname{VAS}\left(r_{s}=-0.488, p<0.05\right)$

\section{DISCUSSION}

This study explored the relationship between the severity of NMS (cognitive impairment, depression, and anxiety), and functional gait and balance performance in people diagnosed with PD. A significant positive correlation was found between the severity of self-reported affective NMS and self-reported physical function. No correlation was observed between the clinician-rated measures of physical (DGI) and cognitive function (MoCA), and self-reported NMS.

NMS are clinically under-recognised in the early stages of the disease, although they have been shown to have greater impact on motor disability and associated QoL (Erro et al., 2016;

Prakash et al., 2016). Understanding the relationship between these constructs can provide direction for future research and clinical management of PD. In the current study, self-reported depression (HADS-D) and anxiety (HADS-A) did not significantly correlate with either clinician-rated physical function (DGI) or clinician-rated motor disability (MDS-UPDRS Part III). These findings differ from those of Ehgoetz-Martens et al. (2016), who found a significant and positive correlation between depression and anxiety with the clinician-rated impression of motor symptom severity (UPDRS Part III). Though not significant in the current study, the strength of association between depression (HADS-D) and motor disability (MDS-UPDRS Part III) was similar to that reported in the previous study (Ehgoetz-Martens et al., 2016). We also observed a fair negative correlation between anxiety (HADS-A) and motor disability (MDS-UPDRS Part III). These differences may be attributable to the motor symptom severity. It is observed that the mean scores of the motor symptom severity were higher in the previous study (EhgoetzMartens et al., 2016) than the current study.

The severity of self-reported depression and anxiety symptoms of participants in our study can be classified as "normal" to "mild", according to the criterion described for the elderly population (Smarr \& Keefer, 2011). Although previous studies with participants of a similar severity of depression (Kincses et al., 2017; Lord et al., 2011; Lord et al., 2013; Rochester et al., 2008) and anxiety (Ehgoetz Martens et al., 2014) demonstrated significant correlations with alterations to gait parameters, our results suggest that changes in gait parameters due to these symptoms may not necessarily determine functional or motor disability. It may be that the level of severity of affective symptoms in our participants was not severe enough to overload the processing resources of the basal ganglia (Gilat et al., 2017) and thus impact functional gait and balance (DGI).

Cognitive impairment (MoCA) had no significant correlation with functional gait and balance (DGI) or motor disability (MDSUPDRS Part II). However, a significant correlation was found between cognitive impairment (MoCA) and clinician-rated motor disability (MDS-UPDRS Part III). These results suggest that cognitive impairment may affect motor symptom severity, but not influence self-reported or clinician-rated impressions of functional mobility. Again, the severity of cognitive impairment in our participants may not have been sufficient to interfere with the increased cognitive demands required to compensate for gait and balance impairments when walking, or the DGI may not have significantly challenged cognitive demands. Thus, insufficient cognitive demand may explain why no correlation with functional gait and balance was shown in this study (Gilat et al., 2017).

Though no correlations were found between functional gait and balance (DGI) with most of the disease specific outcome measures, namely sub-sections of the MDS-UPDRS and disease duration, a significant correlation between functional gait and balance (DGI) with the stage of PD ( $\mathrm{H} \& \mathrm{Y}$ scale) was found. These findings suggest that $D G I$ is sensitive to detect functional gait impairment as rated by the clinicians using the $\mathrm{H} \& \mathrm{Y}$ scale. The current findings suggest that motor symptom severity, and functional gait and balance are influenced by disease stage but not disease duration. This is similar to previous findings on freezing of gait, which reportedly is not linearly related to freezing or festination of gait (Amboni et al., 2008).

The majority of participants were classified as having a "normal" severity of affective symptoms and cognitive function, and no risk of falls. This differs from prevalence studies which have suggested clinically relevant symptoms of depression, anxiety, and mild cognitive impairment in $42 \%$ (Reijnders et al., 2008), $25.7 \%$ (Broen et al, 2016) and up to $21 \%$ of the PD population, respectively (Hobson \& Meara, 2004, 2015; Luck et al., 2010). The cut-off score of DGI for falls risk is 16.3 (Cakit et al., 2007), while our study had a mean DGI score of 18.41

Furthermore, the majority of participants reported no problems $(32 \%-74 \%)$ or only slight problems $(21 \%-58 \%)$ in the EQ-5D dimensions. Participants with moderate problems to extreme disability were underrepresented in this sample. Thus, the external validity of the findings is reduced among those with a greater severity of NMS, and gait and balance impairments with poorer QoL. Our study did, however, include people with a disease stage that ranged from 1 to 4 as evaluated by the $\mathrm{H}$ \& $Y$ scale, with a wide range of self-reported disease duration (11 months to 10 years).

While a generic hypothesis was made for the correlation between NMS and physical function measures, no specific a priori hypotheses were made about the self-reported and clinician-rated measures. However, the findings suggest that the hypothesis was upheld for the self-reported measures but not for the clinician-rated measured. The self-reported measures of affective symptoms and cognition correlated with selfreported motor disability. The clinician-rated physical function did correlate with cognitive impairment but not for other NMS (depression and anxiety). These findings are in line with the functional pathophysiology of PD previously discussed. Our additional hypothesis that NMS severity and functional disability might be correlated with poorer perceived health status (EQVAS) was upheld for depression symptoms but not for anxiety. 
It is understood that the severity of anxiety symptoms, cognitive impairment, and gait and balance impairments may not have been sufficient to influence the health status of the study sample.

\section{Strengths and limitations}

A narrative review published in 2018 suggested the need for further research to better understand the influence of NMS on gait and function in PD (Avanzino et al., 2018). To our knowledge, this is the first study to explore the correlation between the severity of NMS on self-reported and clinicianrated impressions of functional mobility in the PD population. Additionally, to our knowledge, this is the first study to explore the correlation between motor disability and symptom severity as evaluated by the MDS-UPDRS Part II and Part III with a functional gait and balance outcome measure, which we believe is a strength of the study. Another strength of this study is that participants were recruited from people living in the community, thus reducing selection bias. The small sample size ( $n=19$ ) limited our study from being a true representation of characteristics in the PD population. However, bootstrap analysis suggested that the significance of the findings was not limited by the sample size.

The questionnaires used in the study adopted a 7-day recall for any symptoms, which induces the likelihood of recall bias in the self-reported severity - a delimitation of questionnairebased studies. The recall bias that was present may have caused participants to underestimate the severity of their symptoms, and so may explain why most participants were classified as having only "normal" to "mild" symptom severity.

Finally, this study did not compare findings against a control group of healthy age-matched people, nor did it explore the influence of NMS fluctuations on functional gait and balance due the cross-sectional study design used, which is a limitation of the findings. Thus, it remains unclear whether or not NMS severity influences functional gait and balance in the PD population compared to healthy age-matched controls. Furthermore, a longitudinal study may have shown fluctuations in NMS severity to have a stronger association with physical function than symptom severity assessed at one point in time.

Implications

The findings provide preliminary information to suggest that the severity of NMS may be a significant correlation of selfreported functional disability in addition to poorer perception of health status, particularly depressive symptoms. Clinically, the findings point to the need to further explore the relationship between self-reported functional disability and perceived health status with the severity of self-reported NMS. A qualitative investigation exploring the relationship will inform clinicians involved in the management of PD about the use of additional strategies to counter NMS severity and thus improve physical function and QoL. Nevertheless, the findings suggest that the clinician-rated measures may not necessarily reflect the subjective experiences of motor or non-motor experiences. Clinicians should therefore be cognisant of this difference and apply due caution in the interpretation and application of these outcome measure in their practice. Collectively, findings from the current study add to the body of knowledge on the relationship between NMS and physical function, which may help to build future research on PD interventions.

\section{CONCLUSION}

Self-reported functional disability and perceived health status of people with PD appears to correlate with the severity of self-reported NMS. Though no significant correlation was found between self-reported NMS, namely affective symptoms and cognitive impairment, with clinician-rated functional mobility, this possible contention cannot be excluded among people with a greater severity of NMS.

\section{KEY POINTS}

1. There is a relationship between the severity of non-motor symptoms, particularly depressive symptoms, and selfreported functional disability and perceived health status in people with Parkinson's disease (PD).

2. Clinician-rated measures of function may not reflect selfreported experiences of motor or non-motor experiences.

3. PD stage may be a stronger correlate with function than disease duration.

\section{DISCLOSURES}

No funding was received. There are no conflicts of interest which may be perceived to interfere with or bias this study.

\section{PERMISSIONS}

Ethical approval was obtained from the University of Otago Human Ethics Committee (reference number H19/048).

\section{ADDRESS FOR CORRESPONDENCE}

Dr. Prasath Jayakaran, Senior Lecturer, School of Physiotherapy, University of Otago, Dunedin, New Zealand.

Email: prasath.jayakaran@otago.ac.nz

\section{REFERENCES}

Abdolahi, A., Scoglio, N., Killoran, A., Dorsey, E. R., \& Biglan, K. M. (2013). Potential reliability and validity of a modified version of the Unified Parkinson's Disease Rating Scale that could be administered remotely. Parkinsonism \& Related Disorders, 19(2), 218-221. https://doi. org/10.1016/j.parkreldis.2012.10.008

Amboni, M., Cozzolino, A., Longo, K., Picillo, M., \& Barone, P. (2008). Freezing of gait and executive functions in patients with Parkinson's disease. Movement Disorders, 23(3), 395-400. https://doi.org/10.1002/ mds. 21850

Avanzino, L., Lagravinese, G., Abbruzzese, G., \& Pelosin, E. (2018). Relationships between gait and emotion in Parkinson's disease: $A$ narrative review. Gait and Posture, 65, 57-64. https://doi.org/10.1016/j. gaitpost.2018.06.171

Bloem, B. R., Marinus, J., Almeida, Q., Dibble, L., Nieuwboer, A., Post, B. Ruzicka, E., Goetz, C., Stebbins, G., Martinez-Martin, P., \& Schrag, A; for the Movement Disorders Society Rating Scales Committee. (2016). Measurement instruments to assess posture, gait, and balance in Parkinson's disease: Critique and recommendations. Movement Disorders, 31(9), 1342-1355. https://doi.org/10.1002/mds.26572

Bonett, D. G., \& Wright, T. A. (2000). Sample size requirements for estimating Pearson, Kendall and Spearman correlations. Psychometrika, 65(1), 23-28. https://doi.org/10.1007/BF02294183 
Broen, M. P. G., Narayen, N. E., Kuijf, M. L., Dissanayaka, N. N. W., \& Leentjens, A. F. G. (2016). Prevalence of anxiety in Parkinson's disease: A systematic review and meta-analysis. Movement Disorders, 31(8), 11251133. https://doi.org/10.1002/mds. 26643

Cakit, B. D., Saracoglu, M., Genc, H., Erdem, H. R., \& Inan, L. (2007). The effects of incremental speed-dependent treadmill training on postural instability and fear of falling in Parkinson's disease. Clinical Rehabilitation, 21(8), 698-705. https://doi.org/10.1177/0269215507077269

Clark, D. J. (2015). Automaticity of walking: Functional significance, mechanisms, measurement and rehabilitation strategies. Frontiers in Human Neuroscience, 9, 246. https://doi.org/10.3389/fnhum.2015.00246

Craig CL, Marshall AL, Sjostrom M, Bauman, A. E., Booth, M. L., Ainsworth, B. E., Pratt, M., Ekelund, U., Yngve, A., Sallis, J. F., \& Oja, P. (2003). International Physical Activity Questionnaire: 12-country reliability and validity. Medicine and Science in Sports and Exercise, 35(8), 1381-1395. https://doi.org/10.1249/01.MSS.0000078924.61453.FB

Dibble, L. E., \& Lange, M. (2006). Predicting falls in individuals with Parkinson disease: A reconsideration of clinical balance measures. Journal of Neurologic Physical Therapy, 30(2), 60-67. https://doi.org/10.1097/01. npt.0000282569.70920.dc

Ehgoetz Martens, K. A., Ellard, C. G., \& Almeida, Q. J. (2014). Does anxiety cause freezing of gait in Parkinson's disease? PLoS One, 9(9), e106561. https://doi.org/10.1371/journal.pone.0106561

Ehgoetz-Martens, K. A., Hall, J. M., Gilat, M., Georgiades, M. J., Walton, C. C., \& Lewis, S. J. G. (2016). Anxiety is associated with freezing of gait and attentional set-shifting in Parkinson's disease: A new perspective for early intervention. Gait and Posture, 49, 431-436. https://doi.org/10.1016/j. gaitpost.2016.07.182

Erro, R., Picillo, M., Vitale, C., Amboni, M., Moccia, M., Santangelo, G., Pellecchia, M. T. \& Barone, P. (2016). The non-motor side of the honeymoon period of Parkinson's disease and its relationship with quality of life: A 4-year longitudinal study. European Journal of Neurology, 23(11), 1673-1679. https://doi.org/10.1111/ene.13106

Field, A. P. (2009). Discovering statistics using SPSS (3rd ed.). SAGE Publications.

GBD 2015 Neurological Disorders Collaborator Group. (2017). Global, regional, and national burden of neurological disorders during 19902015: A systematic analysis for the Global Burden of Disease Study 2015. Lancet Neurology, 16(11), 877-897. https://doi.org/10.1016/S14744422(17)30299-5

GBD 2016 Neurology Collaborators. (2018). Global, regional, and national burden of Parkinson's disease, 1990-2016: A systematic analysis for the Global Burden of Disease Study 2016. Lancet Neurology, 17(11), 939-953. https://doi.org/10.1016/S1474-4422(18)30295-3

Gilat, M., Bell, P. T., Ehgoetz Martens, K. A., Georgiades, M. J., Hall, J. M., Walton, C. C., Lewis, S. J. G. \& Shine, J. M. (2017). Dopamine depletion impairs gait automaticity by altering cortico-striatal and cerebellar processing in Parkinson's disease. Neurolmage, 152, 207-220. https://doi. org/10.1016/j.neuroimage.2017.02.073

Goetz, C. G., Fahn, S., Martinez-Martin, P., Poewe, W., Sampaio, C., Stebbins, G. T., Stern, M. B., Tilley, B. C., Dodel, R., Dubois, B., Holloway, R., Jankovic, J., Kulisevsky, J., Lang, A. E., Lees, A., Leurgans, S., LeWitt, P. A., Nyenhuis, N., Olanow, C. W., . . LaPelle, N. (2007). Movement Disorder Society-sponsored revision of the Unified Parkinson's Disease Rating Scale (MDS-UPDRS): Process, format, and clinimetric testing plan. Movement Disorders, 22(1), 41-47. https://doi.org/10.1002/mds.21198

Goetz, C. G., Poewe, W., Rascol, O., Sampaio, C., Stebbins, G. T., Counsell, C., Giladi, N., Holloway, R. G., Moore, C. G., Wenning, G. K., Yahr, M. D. \& Seidl, L. (2004). Movement Disorder Society Task Force report on the Hoehn and Yahr staging scale: Status and recommendations The Movement Disorder Society Task Force on rating scales for Parkinson's disease. Movement Disorders, 19(9), 1020-1028. https://doi.org/10.1002/ mds. 20213
Goetz, C. G., Tilley, B. C., Shaftman, S. R., Stebbins, G. T., Fahn, S., MartinezMartin, P., Poewe, W., Sampaio, C., Stern, M. B., Dodel, R., Dubois, B., Holloway, R., Jankovic, J., Kulisevsky, J., Lang, A. E., Lees, A., Leurgans, S., LeWitt, P. A., Nyenhuis, D., . . LaPelle, N. (2008). Movement Disorder Society-sponsored revision of the Unified Parkinson's Disease Rating Scale (MDS-UPDRS): Scale presentation and clinimetric testing results. Movement Disorders, 23(15), 2129-2170. https://doi.org/10.1002/ mds. 22340

Heesch, K. C., van Uffelen, J. G., Hill, R. L., \& Brown, W. J. (2010). What do IPAQ questions mean to older adults? Lessons from cognitive interviews. The International Journal of Behavioral Nutrition and Physical Activity, 7, 35. https://doi.org/10.1186/1479-5868-7-35

Herman, T., Inbar-Borovsky, N., Brozgol, M., Giladi, N., \& Hausdorff, J. M. (2009). The Dynamic Gait Index in healthy older adults: The role of stair climbing, fear of falling and gender. Gait and Posture, 29(2), 237-241. https://doi.org/10.1016/j.gaitpost.2008.08.013

Hinnell, C., Hurt, C. S., Landau, S., Brown, R. G., Samuel, M.; on behalf of the PROMS-PD Study Group. (2012). Nonmotor versus motor symptoms: How much do they matter to health status in Parkinson's disease? Movement Disorders, 27(2), 236-241. https://doi.org/10.1002/mds.23961

Hobson, P., \& Meara, J. (2004). Risk and incidence of dementia in a cohort of older subjects with Parkinson's disease in the United Kingdom. Movement Disorders, 19(9), 1043-1049. https://doi.org/10.1002/mds.20216

Hobson, P., \& Meara, J. (2015). Mild cognitive impairment in Parkinson's disease and its progression onto dementia: A 16-year outcome evaluation of the Denbighshire cohort. International Journal of Geriatric Psychiatry 30(10), 1048-1055. https://doi.org/10.1002/gps.4261

Huang, S.-L., Hsieh, C.-L., Wu, R.-M., Tai, C.-H., Lin, C.-H., \& Lu, W.-S. (2011). Minimal detectable change of the Timed "Up \& Go" Test and the Dynamic Gait Index in people with Parkinson disease. Physical Therapy, 91(1), 114-121. https://doi.org/10.2522/ptj.20090126

Khan, M. A., Quadri, S. A., \& Tohid, H. (2017). A comprehensive overview of the neuropsychiatry of Parkinson's disease: A review. Bulletin of the Menninger Clinic, 81(1), 53-105. https://doi.org/10.1521/ bumc.2017.81.1.53

Kincses, P., Kovács, N., Karádi, K., Feldmann, Á., Dorn, K., Aschermann, Z., Komoly, S., Szolcsányi, T., Csathó, Á., \& Kállai, J. (2017). Association of gait characteristics and depression in patients with Parkinson's disease assessed in goal-directed locomotion task. Parkinson's Disease, 2017 6434689. https://doi.org/10.1155/2017/6434689

Lai, M. H. C. (2020). Bootstrap confidence intervals for multilevel standardized effect size. Multivariate Behavioral Research. Advance online publication. https://doi.org/10.1080/00273171.2020.1746902

Leentjens, A. F. G., Dujardin, K., Marsh, L., Richard, I. H., Starkstein, S. E., \& Martinez-Martin, P. (2011). Anxiety rating scales in Parkinson's disease: A validation study of the Hamilton anxiety rating scale, the Beck anxiety inventory, and the hospital anxiety and depression scale. Movement Disorders, 26(3), 407-415. https://doi.org/10.1002/mds.23184

Lewis, S. J. G., \& Barker, R. A. (2009). A pathophysiological model of freezing of gait in Parkinson's disease. Parkinsonism \& Related Disorders, 15(5), 333-338. https://doi.org/10.1016/j.parkreldis.2008.08.006

Lord, S., Baker, K., Nieuwboer, A., Burn, D., \& Rochester, L. (2011). Gait variability in Parkinson's disease: An indicator of non-dopaminergic contributors to gait dysfunction? Journal of Neurology, 258, 566-572. https://doi.org/10.1007/s00415-010-5789-8

Lord, S., Galna, B., Coleman, S., Burn, D., \& Rochester, L. (2013). Mild depressive symptoms are associated with gait impairment in early Parkinson's disease. Movement Disorders, 28(5), 634-639. https://doi. org/10.1002/mds.25338 
Luck, T., Riedel-Heller, S. G., Luppa, M., Wiese, B., Wollny, A., Wagner, M., Bickel, H., Weyerer, M., Pentzek, M., Moesch, E., Werle, J., Eisele, M., Maier, W., Van Den Bussche, H., Kaduszkiewicz, H., for the AgeCoDe Study Group. (2010). Risk factors for incident mild cognitive impairment - results from the German Study on Ageing, Cognition and Dementia in Primary Care Patients (AgeCoDe). Acta Psychiatrica Scandinavica, 121(4), 260-272. https://doi.org/10.1111/j.1600-0447.2009.01481.x

Mak, M. K., Wong-Yu, I. S., Shen, X., \& Chung, C. L. (2017). Long-term effects of exercise and physical therapy in people with Parkinson disease. Nature Reviews. Neurology, 13(11), 689-703. https://doi.org/10.1038/ nrneurol.2017.128

Martinez-Martin, P., Jeukens-Visser, M., Lyons, K. E., Rodriguez-Blazquez, C., Selai, C., Siderowf, A., Welsh, M., Poewe, W., Rascol, O., Sampaio C., Stebbins, G. T., Goetz, C. G., \& Schrag, A. (2011). Health-related quality-of-life scales in Parkinson's disease: Critique and recommendations. Movement Disorders, 26(13), 2371-2380. https://doi.org/10.1002/ mds. 23834

Nasreddine, Z. S., Phillips, N. A., Bédirian, V., Charbonneau, S., Whitehead, V., Collin, I., Cummings, J. L., \& Chertkow, H. (2005). The Montreal Cognitive Assessment, MoCA: A brief screening tool for mild cognitive impairment. Journal of the American Geriatrics Society, 53(4), 695-699. https://doi.org/10.1111/j.1532-5415.2005.53221.x

Peterson, D. S., \& Horak, F. B. (2016). Neural control of walking in people with Parkinsonism. Physiology, 31(2), 95-107. https://doi.org/10.1152/ physiol.00034.2015

Pitcher, T. L., Myall, D. J., Pearson, J. F., Lacey, C. J., Dalrymple-Alford, J. C., Anderson, T. J., \& MacAskill, M. R. (2018). Parkinson's disease across ethnicities: A nationwide study in New Zealand. Movement Disorders, 33(9), 1440-1448. https://doi.org/10.1002/mds.27389

Portney, L. G., \& Watkins, M. P. (2015). Foundations of clinical research: Applications to practice (3rd ed.). Pearson/Prentice Hall

Prakash, K. M., Nadkarni, N. V., Lye, W.-K., Yong, M.-H., \& Tan, E.-K. (2016). The impact of non-motor symptoms on the quality of life of Parkinson's disease patients: A longitudinal study. European Journal of Neurology, 23(5), 854-860. https://doi.org/10.1111/ene.12950

Reijnders, J. S. A. M., Ehrt, U., Weber, W. E. J., Aarsland, D., \& Leentjens, A. F. G. (2008). A systematic review of prevalence studies of depression in Parkinson's disease. Movement Disorders, 23(2), 183-189. https://doi. org/10.1002/mds. 21803
Rocca, W. A. (2018). The burden of Parkinson's disease: A worldwide perspective. Lancet Neurology, 17(11), 928-929. https://doi.org/10.1016/ S1474-4422(18)30355-7

Rochester, L., Nieuwboer, A., Baker, K., Hetherington, V., Willems, A.-M., Kwakkel, G., Van Wegen, E., Lim, I., \& Jones, D. (2008). Walking speed during single and dual tasks in Parkinson's disease: Which characteristics are important? Movement Disorders, 23(16), 2312-2318. https://doi. org/10.1002/mds.22219

Rossi, A., Berger, K., Chen, H., Leslie, D., Mailman, R. B., \& Huang, X. (2018). Projection of the prevalence of Parkinson's disease in the coming decades: Revisited. Movement Disorders, 33(1), 156-159. https://doi.org/10.1002/ mds. 27063

Schrag, A., Jahanshahi, M., \& Quinn, N. (2000). How does Parkinson's disease affect quality of life? A comparison with quality of life in the general population. Movement Disorders, 15(6), 1112-1118. https://doi. org/10.1002/1531-8257(200011)15:6<1112::Aid-mds1008>3.0.Co;2-a

Skorvanek, M., Goldman, J. G., Jahanshahi, M., Marras, C., Rektorova, I., Schmand, B., van Duijn. E., Goetz, C. G., Weintraub, D., Stebbins, G. T., Martinez-Martin, P., \& members of the MDS Rating Scales Review Committee. (2018). Global scales for cognitive screening in Parkinson's disease: Critique and recommendations. Movement Disorders, 33(2), 208-218. https://doi.org/10.1002/mds.27233

Smarr, K. L., \& Keefer, A. L. (2011). Measures of depression and depressive symptoms: Beck Depression Inventory-II (BDI-II), Center for Epidemiologic Studies Depression Scale (CES-D), Geriatric Depression Scale (GDS), Hospital Anxiety and Depression Scale (HADS), and Patient Health Questionnaire-9 (PHQ-9). Arthritis Care and Research, 63(S11), S454S466. https://doi.org/10.1002/acr.20556

Soh, S.-E., Morris, M. E., \& McGinley, J. L. (2011). Determinants of healthrelated quality of life in Parkinson's disease: A systematic review. Parkinsonism \& Related Disorders, 17(1), 1-9. https://doi.org/10.1016/j. parkreldis.2010.08.012

Zigmond, A. S., \& Snaith, R. P. (1983). The hospital anxiety and depression scale. Acta Psychiatrica Scandinavica, 67(6), 361-370. https://doi. org/10.1111/j.1600-0447.1983.tb09716.x 